Cite this: Soft Matter, 2014, 10, 4789

\title{
Buckling of dielectric elastomeric plates for soft, electrically active microfluidic pumps
}

Received 4th April 2014

Behrouz Tavakol, ${ }^{a}$ Michael Bozlar, ${ }^{b}$ Christian Punckt, ${ }^{\text {b }}$ Guillaume Froehlicher, ${ }^{c}$ Howard A. Stone, ${ }^{c}$ Ilhan A. Aksay ${ }^{b}$ and Douglas P. Holmes ${ }^{\star a}$

Accepted 14th May 2014

DOI: $10.1039 / \mathrm{c} 4 \mathrm{sm} 00753 \mathrm{k}$

www.rsc.org/softmatter

Elastic instabilities, when properly implemented within soft, mechanical structures, can generate advanced functionality. In this work, we use the voltage-induced buckling of thin, flexible plates to pump fluids within a microfluidic channel. The soft electrodes that enable electrical actuation are compatible with fluids, and undergo large, reversible deformations. We quantified the onset of voltage-induced buckling, and measured the flow rate within the microchannel. This embeddable, flexible microfluidic pump will aid in the generation of new stand-alone microfluidic devices that require a tunable flow rate.

Advances in microfluidic technology have introduced innovative ways to control fluid flow on a small scale. ${ }^{1-5}$ The active control of fluid flow within such devices is crucial for further improvements in nanofluidics, ${ }^{6,7}$ biomedical fluidic devices, ${ }^{8-10}$ and digital microfluidics. ${ }^{11-13}$ The complexity of microfluidic channels has advanced to resemble integrated circuitry, ${ }^{\mathbf{1 , 4 1 4}}$ and the mechanisms that move fluid within these channels now require the same degree of flexibility and precision. Electrically active soft materials that deform in response to an applied voltage may provide this advanced functionality. ${ }^{15} \mathrm{In}$ this paper, we present a means for microfluidic control via the electrical actuation of thin, flexible films within microfluidic channels. These structures consist of a dielectric elastomer confined between two compliant electrodes that can actively and reversibly buckle out of the plane to pump fluids in response to an applied voltage. The use of elastic electrodes enables a robust and reversible pumping mechanism that will have improvements in rapid microfluidic diagnostics, adaptive materials, and artificial muscles.

${ }^{a}$ Department of Engineering Science \& Mechanics, Virginia Tech, Blacksburg, VA 24061, USA. E-mail: dpholmes@vt.edu; Fax: +1 (540) 231-4574; Tel: +1 (540) 2317814

${ }^{b}$ Department of Chemical \& Biological Engineering, Princeton University, Princeton, NJ 08544, USA

'Department of Mechanical \& Aerospace Engineering, Princeton University, Princeton, NJ 08544, USA
When a film of a soft dielectric elastomer (DE) is sandwiched between two electrode films, creating a composite plate (Fig. 1a), applying a voltage to the electrodes produces an electric field within the DE that induces a Maxwell stress through its thickness. ${ }^{16}$ As a result, the DE is compressed by pulling opposite charges on the two electrodes closer and stretched by spreading similar charges on each electrode. ${ }^{17,18}$ If the composite plate has free edges, the applied voltage will cause it to expand in-plane. ${ }^{19-25}$ If, instead, the plate is clamped at the electrode edges, thus prevented from expanding laterally, the plate will buckle out of the plane above a critical compressive stress. In this paper, we show that embedding this confined plate within a microfluidic channel and applying an adequate voltage (Fig. 1b) allows the buckling instability to move the fluid and control the flow (Fig. 1c). We will discuss the critical voltage required to induce buckling, the subsequent buckling modes that emerge, and the impact that the pressure around the electro-active plate has on the fluid flow rate.
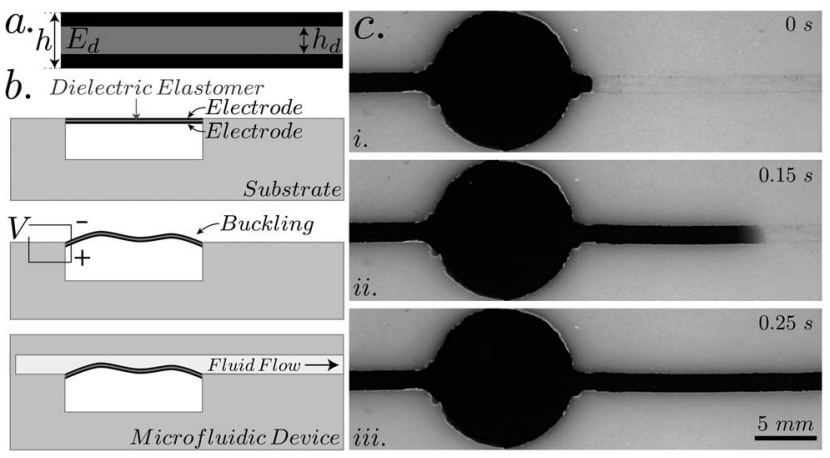

Fig. 1 (a) A schematic of the composite plate. (b) Schematics of the electrically active microfluidic pump: a thin composite plate consists of a prestrained dielectric elastomer with electrodes bonded on each side while the plate is clamped at its edges. Applying a voltage across the clamped thin plate causes an out-of-plane deformation, which can be used to move the fluid within microchannels. (c) Actuation of the dielectric elastomer by applying the voltage pushes fluid (dyed water) with a flow rate of $20 \mathrm{~mm}^{3} \mathrm{~s}^{-1}$ into the microchannel. 

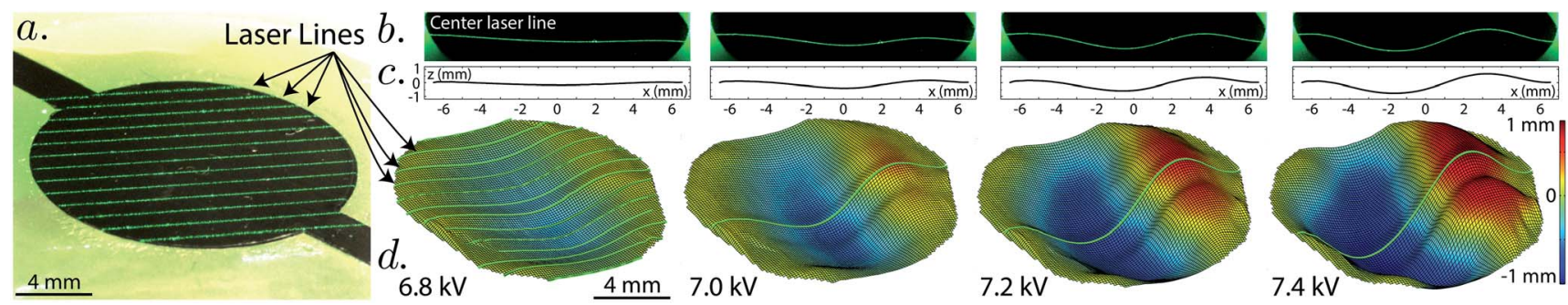

Fig. 2 3D surface reconstruction of the shape of the thin plate by taking and processing the images of different cross sections at different voltages. (a) Overlaid images of the laser line at different cross sections when the voltage is zero. (b) Raw images of the laser line for the middle cross section taken at different voltages. (c) Extracted profiles of the images shown in part (b) using image processing. (d) 3D surface profile of the thin plate at different voltages.

The composite plate is prepared by biaxially stretching the DE and then bonding the solid electrodes to it. In addition to a significant thickness reduction which eventually lowers the actuation voltage, the prestrain mechanism improves the DE's stability against different failure modes, as well as the voltage breakdown characteristics of this material. ${ }^{24}$ The prestrain also leaves a residual stress $\sigma_{\mathrm{i}}$ in the DE. Applying a voltage $V$ to the electrodes, which are clamped along their edges, induces a compressive Maxwell stress $\sigma_{\mathrm{e}}$ through the DE thickness, which initially reduces $\sigma_{\mathrm{i}}$. Once the total radial stress, $\sigma_{\mathrm{r}}=\sigma_{\mathrm{e}}-\sigma_{\mathrm{i}}$, exceeds the critical buckling stress for the clamped circular plate, it will deform out of the plane.

To examine the topography of the buckled plate, a laser line was imaged by a camera to record the deformations of the circular plate at different locations on its surface (Fig. 2a). The lines remain unperturbed until the applied voltage generates a stress that exceeds the critical buckling stress of the plate. As the plate deforms out of the plane (Fig. 2b), we extract the deformation profiles of each laser line via image processing (Fig. 2c) to obtain a quantitative measure of the entire deformation of the plate (Fig. 2d).

We determined the onset of buckling by measuring how the length of the line $L$ along the diameter changes as a function of $V$. We normalized $L$ by the initial length of the cross section, $L_{0}$, and plotted it versus applied voltage in Fig. 3a. The results show a sharp increase in $L$, corresponding to an out-of-plane deformation around $V=6.8 \mathrm{kV}$. Changing the conditions surrounding the composite plate, such as the pressure above and below it (Fig. 3b), has a dramatic effect on the post-buckled shape of the plate (Fig. 3c), yet they appear to have no effect on the onset of buckling (Fig. 3a).

We first seek to describe the onset of buckling when a voltage is applied to the thin plate. The relation between $V$ and the radial strain $e_{\mathrm{r}}$ for a free-standing, thin plate is described by:

$$
e_{\mathrm{r}} \approx \frac{\varepsilon_{0} \varepsilon}{2 E_{\mathrm{d}}}\left(\frac{V}{h_{\mathrm{d}}}\right)^{2}
$$

where $E_{\mathrm{d}}$ is the elastic modulus of the $\mathrm{DE}, \varepsilon_{0}$ is the permittivity constant, $\varepsilon$ is the relative permittivity of the DE, $h_{\mathrm{d}}$ is the thickness of the dielectric layer, and $V$ is the applied voltage. ${ }^{17}$ We assume that near the buckling threshold the material is incompressible and elastic, therefore the applied voltage causes a stress in the radial direction in the form $\sigma_{\mathrm{e}} \approx \frac{\varepsilon_{0} \varepsilon}{2\left(1-\nu^{2}\right)}\left(V / h_{\mathrm{d}}\right)^{2}$, where $\nu \approx 0.5$ is the Poisson ratio of the DE. At the onset of buckling, the deflection of the plate is small relative to the plate thickness, so we use the linearized plate equations as an estimation of the critical buckling stress. A clamped, circular plate exposed to a radial compressive stress will buckle out of the plane when $\left(\sigma_{\mathrm{r}}\right)_{\mathrm{cr}}=\frac{k D}{R^{2} h}$, where $R$ is the plate's radius, $D=\frac{E h^{3}}{12\left(1-\nu^{2}\right)}$ is the flexural rigidity, and $k$

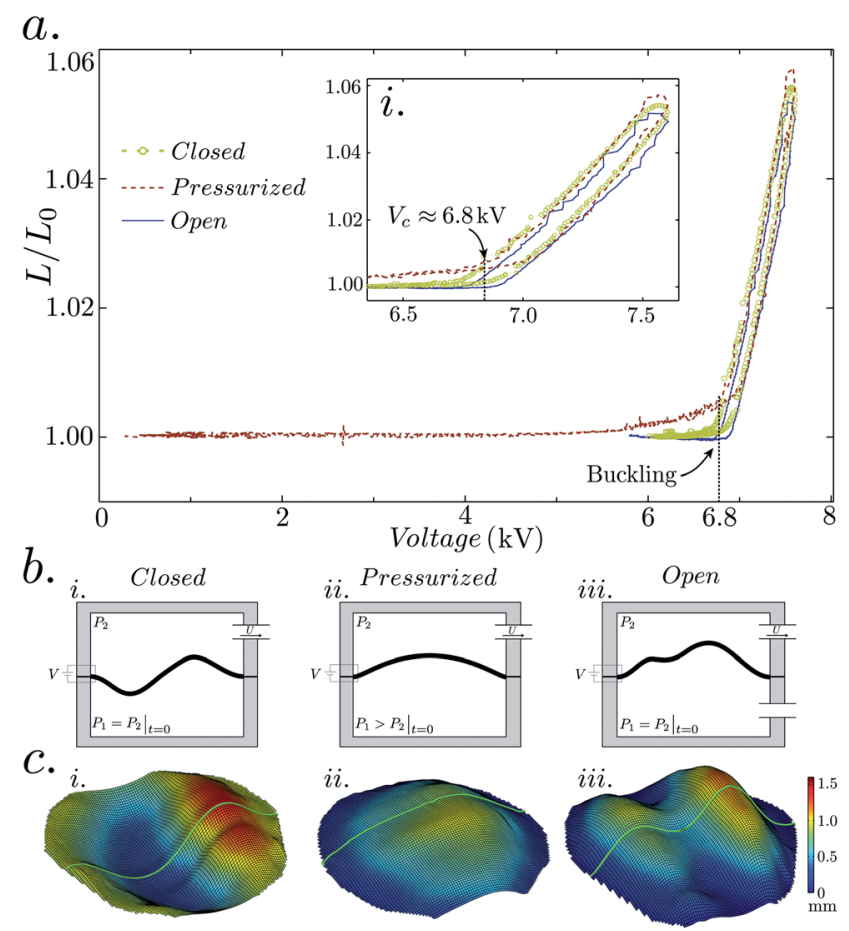

Fig. 3 (a) Changes in line length of the middle cross section normalized with the initial line length at zero voltage. The inset (same units) shows occurrence of the critical buckling voltage at which the line length starts changing linearly with the voltage. This critical buckling threshold appears to be independent of the pressure in the surrounding chambers. (b and c) Schematics and corresponding 3D profiles for the three modes of electrically induced deformation by varying volume and pressure above and below the thin plate: (i) closed. One chamber is closed but both chambers initially have the same pressure, (ii) pressurized. One chamber is closed and at a higher pressure (iii) open. Both sides are open and have the same pressure. 
is a numerical factor that depends on the boundary conditions and the buckling mode; $k_{1}=14.68$ and $k_{2}=26.4$ for modes 1 and 2 of a clamped plate, respectively. ${ }^{26}$ Considering both the residual stress and the voltage-induced stress, we estimate the critical voltage for plate buckling as:

$$
V_{\mathrm{c}} \approx h_{\mathrm{d}}\left(\frac{k E}{6 \varepsilon_{0} \varepsilon}\left(\frac{h}{R}\right)^{2}+\frac{2\left(1-\nu^{2}\right) \sigma_{\mathrm{i}}}{\varepsilon_{0} \varepsilon}\right)^{1 / 2}
$$

which is similar to linearized critical voltage equations reported in other studies. ${ }^{27}$ We consider the effect of prestrain as an initial condition for this system, and use material properties measured under the applied prestrain. We expect that small deformations should result in a linear behavior, and therefore, this linearized theory will be accurate in describing the onset of buckling. $\dagger$ When $h / R \ll 1$, the critical voltage is dominated by the residual stress. For example, in our experiments $h / R \approx 0.03$, so the experimentally measured residual stress within the plate, $\sigma_{\mathrm{i}}=\mathscr{O}\left(10^{2} \mathrm{kPa}\right)$, is significantly larger than the stress required to buckle an initially stress-free plate, $\sigma_{\mathrm{c}}=\mathscr{O}(1 \mathrm{kPa})$. Since the applied voltage in this case mainly serves to reduce the residual stress in the dielectric film, we approximate the critical voltage by considering only the residual stress term. Neglecting numerical factors of order one, we find that for a thin, prestrained film with significant residual stress,

$$
V_{\mathrm{c}} \sim \sqrt{\frac{h_{\mathrm{d}}^{2} \sigma_{\mathrm{i}}}{\varepsilon_{0} \varepsilon}} .
$$

Eqn (3) predicts that the critical voltage for the composite plates shown in Fig. 3 should be around $V_{\mathrm{c}}=(4.8 \pm 0.9) \mathrm{kV}$, which deviates by about $25 \%$ from our experimentally observed value of $V_{\mathrm{c}} \approx 6.8 \mathrm{kV}$. Also, eqn (3) suggests that the critical voltage should scale linearly with the film thickness. Experiments over a wide range of $h$ will be necessary to verify this scaling, though this work is beyond the scope of the current article.

While the above calculation is useful for determining the critical threshold for buckling, it says nothing about the postbuckled shape of the plate. The difference between the pressure and volume above and below the plate will control the postbuckled shape, which will, in turn, determine the structure's ability to move fluid within the microchannel. We use the imaging technique demonstrated in Fig. 2 to determine both the plate's buckling mode, and the expected fluid flow rate. Since the plate resides in a chamber of known dimensions (Fig. 3b), integration of the volume above or below the 3D surfaces in Fig. 3c allows us to define a geometrical flow rate, which gives a measure of the net volume change as a function of time for a given voltage. By assuming the flow is incompressible, this net volume change yields the fluid flow rate as a function of voltage. This geometrically derived flow rate was verified using both a flow meter and optical imaging. To determine the effect of the chamber pressure on the postbuckled plate's deformation, we embedded the plate in a controlled environment, and varied the surrounding pressure. We identified three important regimes that determine the plate's deformation.
First, both sides of the plate are exposed to a constant pressure $P_{0}$, and reside within a closed chamber (Fig. 3b-i). In this case, a mode two, asymmetric out-of-plane deformation was observed once the critical buckling threshold was reached (Fig. 3c-i). The amplitude of the deformations increased with the voltage, and the mode two shape remained well into the post-buckling regime. Since this shape has an up-down symmetry about the horizontal axis, it does not move any fluid in the top chamber. Accordingly, both the geometrical flow rate and the experimental flow rate from the flow meter measured zero flow (Fig. 4a). This buckling mode can be easily understood by considering that the plate's surface area increases with the applied voltage, but any axisymmetric deformation would cause the chamber below the plate to be pressurized. Therefore, the minimal energy corresponds to an out-of-plane deformation with up-down symmetry, which will have a negligible change on the pressure in the closed chamber. To understand this effect, we consider that the bending energy of the plate scales as $U_{\mathrm{b}} \sim$ $E h^{3} \kappa^{2}$, where $\kappa$ is the curvature, and therefore the work done by bending has the form $W_{\mathrm{b}} \sim E h^{3} R \kappa$. The work done by the pressure is $W_{\mathrm{p}}=P^{\mathscr{V}}$, where $\mathscr{V}$ is the volume change above or below the plate, which we estimate from the volume of a spherical cap $\mathscr{V}=(3 R-w) \pi w^{3} / 3 \sim R^{4} \kappa$ for a small cap height of $w$. The ratio of $W_{\mathrm{b}} / W_{\mathrm{p}} \sim(E / P)(h / R)^{3} \sim 10^{-6}$ indicates that

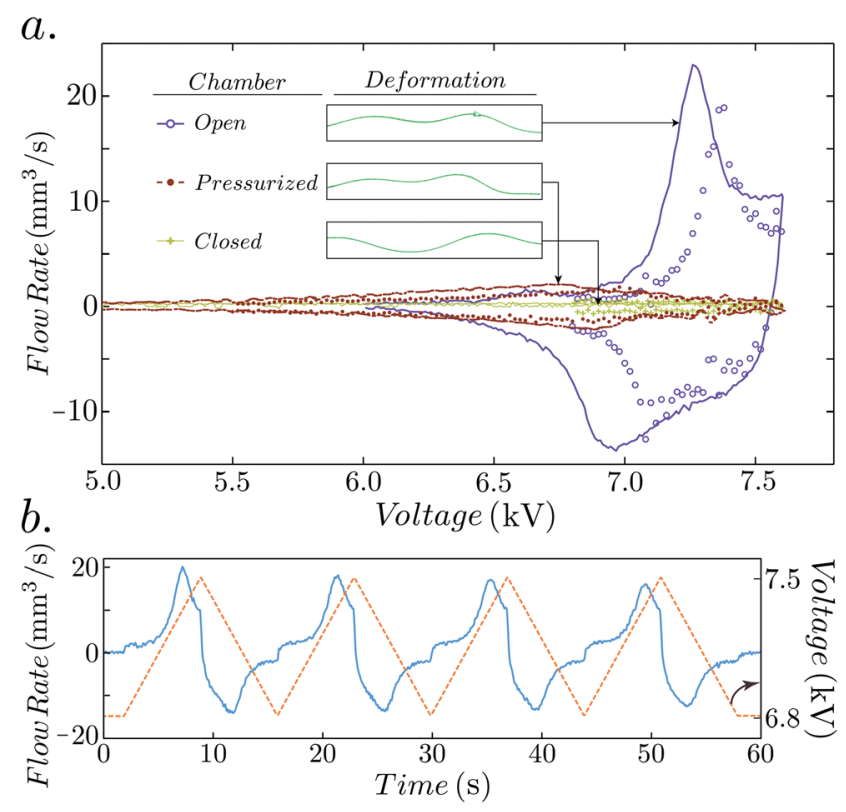

Fig. 4 (a) Flow rate as a function of voltage for different cases measured using a flow meter (lines) and estimated geometrically from 3D surface profiles (dots). The voltage was applied at a rate of $100 \mathrm{~V} \mathrm{~s}^{-1}$ for all cases. The flow rate of the 'open' case was an order of magnitude higher than the one of the 'pressurized' case while the 'closed' case resulted in zero flow rate. (b) Cyclic flow rate of the pump, measured using a flow meter, for the 'open' case when the voltage stimulus was in the form of a triangle waveform oscillating between $6.8 \mathrm{kV}$ and $7.5 \mathrm{kV}$. The pump showed a repeatable flow rate profile and the slight decrease in the maximum flow rate of different cycles can be related to viscoelastic effects of the plate. Note that for each cycle, we have a pumping mechanism followed by a suction that is useful for the pumping in the next cycle. 
buckling of a thin plate is energetically more favorable than changing the pressure in the enclosed chambers. Therefore, the plate will spontaneously adopt a higher mode of buckling that does not necessitate a change in pressure in the surrounding chambers.

Second, we prescribe one chamber to be at a higher pressure than the other, i.e. $P>P_{0}$. A mode one, axisymmetric out-ofplane deformation was observed above the buckling threshold (Fig. 3b-ii). We applied a positive initial pressure of $P \approx 500 \mathrm{~Pa}$ to the bottom channel and increased the voltage linearly at a rate of $100 \mathrm{~V} \mathrm{~s}^{-1}$ to $7.6 \mathrm{kV}$. We observed a small deformation before reaching $6.8 \mathrm{kV}$, which has also been reported in previous studies; $;^{28,29}$ however, the thin film underwent significant deformation when the voltage exceeded $6.8 \mathrm{kV}$. The axisymmetry of the buckled plate, enabled by the pressure difference between the two chambers, results in a net positive flow out of the open chamber. We measured a flow rate that increases slowly with voltage until $6.8 \mathrm{kV}$, followed by a decrease afterward (Fig. 4a).

Finally, we consider the case when both channels are open and exposed to air, so the pressure difference between two sides of the thin film is zero (Fig. 3b-iii). In this case, a highly nonlinear, yet reproducible, shape emerged at the onset of buckling, and varied with increasing applied voltage. Exceeding $7.4 \mathrm{kV}$ caused the entire thin plate to rapidly undergo a snapthrough to compensate the further surface extension. These factors lead to a significant flow rate, at least one order of magnitude higher than the other two cases (Fig. 4a). We suspect this response is because the deformation of the thin film is not restricted by changes in pressure of each chamber. Further study of coupled interactions between the fluid and the flexible plate may be necessary to fully characterize the effect of pressure on the buckling dynamics, which will be left as future work.

Both the shape of the buckled plate and the resulting fluid flow rate are dependent on the pressures in the chambers above and below it. Positive, directional flow is observed when one chamber is pressurized, or when both chambers are open to the atmosphere. Incorporating these concepts into the design of a microfluidic system provides a voltage-induced means for generating flow. As a demonstration of this pumping mechanism, we applied a voltage cycle and measured the flow rate as a function of time (Fig. $4 \mathrm{~b}$ ), which produced both pumping and suction within the channel.

Current microfluidic designs involve closed chambers with equal pressures - matching the scenario in which no flow was measured. To address this limitation, we provide a simple solution for generating flow by coupling multiple pumps. We consider two pumps that are connected in series, i.e. each pump is connected to one end of a microfluidic channel. The bottom chambers of the pumps are also connected through the "controlling" channel, to couple the pumping action (Fig. 5a). Since we have a constant volume in the bottom channels, each pump operates similar to a single pump with a closed chamber and same initial pressure, where the flow rate was zero. By applying the voltage, both plates buckle, but the deformation of one affects the deformation of the other. In fact, plates deform by pulling/pushing the air from one chamber to the next one
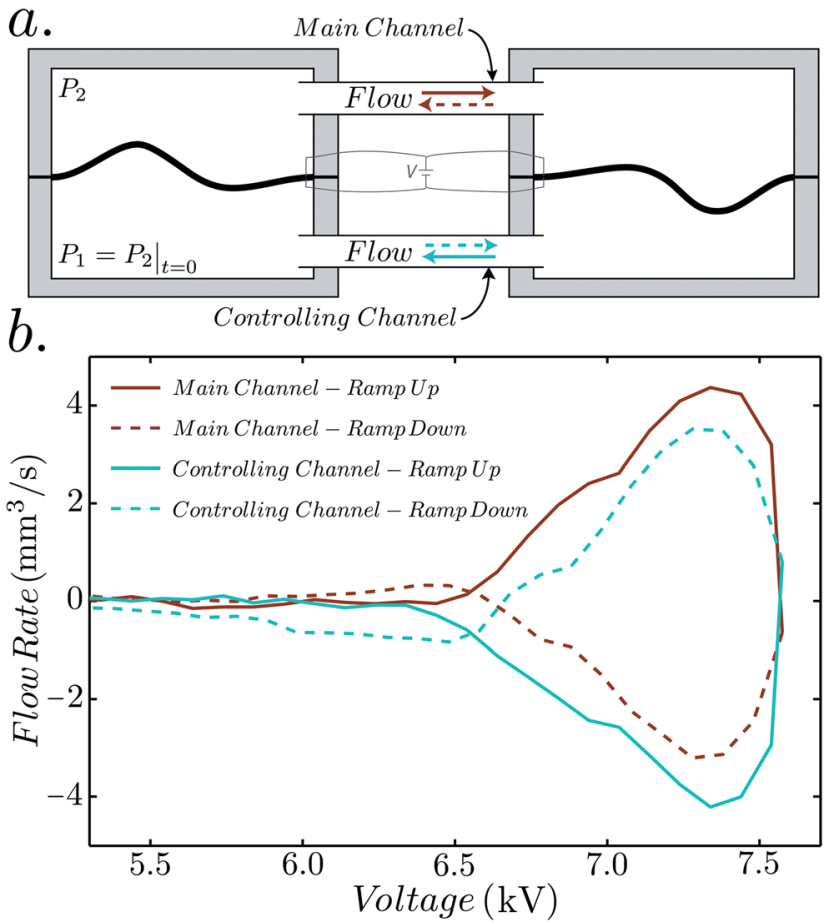

Fig. 5 (a) A schematic of combining two pumps in series; bottom channels are also connected to enhance the pumping action. (b) When the left plate deforms, air is pulled out from the bottom channel from the right to the left pump, forcing the right plate to deform in the opposite direction and therefore pulling the air in the top channel in the reverse direction, from the left to the right and increasing the efficiency of pumping. This mechanism is reversed when decreasing the voltage to zero. The rate of change of voltage is $100 \mathrm{~V} \mathrm{~s}^{-1}$.

without a need for changing the volume or pressure of the surrounding medium. Therefore, we observe higher flow rates due to the coupling of suction and pumping between these two pumps (Fig. 5b). This effect is reversed when the voltage is decreased, providing a robust means for controllable bidirectional flows. Similar bidirectional flows have been observed in the heartbeat mechanism of the dorsal vessel in some insects, e.g. aperygotes and mayflies. ${ }^{30}$ Note that this is a closed and isolated system where outside pressure does not play any role in plate deformation, so the flow rate of this system is most comparable to the one of a 'closed' pump discussed above, which shows a significant increase in the flow rate. The versatility of the advanced material design that we present allows these pumps in series or in parallel to enable bidirectional flows and microfluidic vacuum pumps with enhanced flow rates.

In conclusion, we utilized the voltage-induced out-of-plane deformation of a confined dielectric thin plate as a means to pump fluids within microchannels. We prepared elastic electrodes that are flexible, and can be in direct contact with fluid. In addition to the applied voltage, we considered the effect of pressure and volume on the deformation of the thin plate. The change in surface area depends on the voltage, while the deformation shape, which significantly affects the flow rate, is a function of voltage and pressure. The flow rate was observed to be as high as $20 \mathrm{~mm}^{3} \mathrm{~s}^{-1}$. These pumps can also be utilized in series and/or parallel in order to enhance the flow rate, or add 
advanced functionality such as microchannel vacuums or bidirectional fluid flow. These steps will open new avenues for microfluidic systems where a low power consumption pump with a tunable flow rate can easily be integrated.

\section{Experimental}

\section{Preparation of conductive thin films and composite thin plate}

VHB 4910 acrylic tape (3 M) was used as the dielectric elastomeric film, which was stretched biaxially to $250 \%$ prestrain, and attached to an acrylic frame (8560K171, McMaster-Carr) for maintaining the prestrain. Flexible conductive electrodes were made by mixing carbon black (CB) particles into PDMS using the following procedure: $3 \mathrm{wt} \% \mathrm{CB}$ particles (Ketjenblack EC-600JD, AkzoNobel) were dispersed in $40 \mathrm{~mL}$ of tetrahydrofuran (Sigma-Aldrich) for 1 hour using a tip-ultrasonicator (VirSonic 100). An ice-bath was used to prevent overheating of the suspension. Then, the suspension was transferred to the uncrosslinked PDMS (vinyl terminated PDMS with 9400 Da molecular weight, Gelest Inc.) that was preheated to $70{ }^{\circ} \mathrm{C}$. The mixture was continuously stirred and heated for about 4 hours to allow the solvent to evaporate. After adding $22 \mathrm{mg}$ of the catalyst (platinum-cyclovinylmethylsiloxane complex, Gelest Inc.) and $99 \mathrm{mg}$ of the crosslinker (tetrakis(dimethylsiloxy) silane, Gelest Inc.), and manual mixing for 10 minutes, the mixture was cast directly on both sides of the prestrained DE film. The geometry of the conducting PDMS electrodes was defined using a polyester film with a circular pattern (diameter: $13.5 \mathrm{~mm}$ ). Electrodes cast on DE films were cured at room temperature for 48 hours prior to use. To easily apply voltage to the electrodes, electrical connections were made with conductive copper tape (SPI Supplies) bonded to the conducting PDMS electrodes using a silver epoxy paste (CW2400, Ted Pella Inc.).

\section{Characterization of the composite thin plate}

The geometrical parameters and material properties of the fabricated pumping device are as follows: plate radius was measured using a caliper $(R=6.75 \mathrm{~mm})$. DE thickness was calculated based on the applied pre-strain $\left(h_{\mathrm{d}}=(82 \pm 5) \mu \mathrm{m}\right)$. The plate's total thickness was measured using a caliper to be $h$ $=(180 \pm 20) \mu \mathrm{m}$. To determine the residual stress, we applied constant $250 \%$ strain to VHB samples with thickness of $1 \mathrm{~mm}$, gauge length of $128 \mathrm{~mm}$, and width of $25 \mathrm{~mm}$, and measured the changes in force under that strain for $30 \mathrm{~min}$. Uniaxial residual stress was then obtained by dividing the plateau value of the force curve by the final cross sectional area of the samples $\left(\sigma_{\mathrm{i}}=(100 \pm 40) \mathrm{kPa}\right)$. After running the stress relaxation test and while the sample was under $250 \%$ strain, we performed a simple tension test to obtain the elastic modulus of the VHB film under prestrain and subjected to stress relaxation condition $\left(E_{\mathrm{d}}=350 \mathrm{kPa}\right)$. Electrode modulus was $E_{\mathrm{e}}=800 \mathrm{kPa}$ (ref. $19)$ and we used rules of mixtures to estimate the plate's effective modulus $(E=544 \mathrm{kPa})$. The permittivity constant is $\varepsilon_{0}=$ $8.85 \times 10^{-12} \mathrm{~Pa} \mathrm{~m}^{2} \mathrm{~V}^{-2}$ and the relative permittivity of VHB is reported as $\varepsilon=3.21 .^{19}$

\section{Fabrication of the microfluidic pump device}

The bottom substrate was made of polyvinylsiloxane (PVS) (Elite double 32, Zhermack) and molded accordingly to define a cylindrical chamber matching the diameter of the conductive PDMS electrodes. Then, a thin layer of uncrosslinked PVS was manually spread on the surface of the bottom substrate and the thin plate was placed on top to chemically bond the bottom substrate and the entire film except the circular actuator. Similarly, the top substrate with a cylindrical chamber and a micro-sized channel $(h=\mathscr{O}(50 \mu \mathrm{m}))$ was made from PVS and chemically bonded to the other side of the thin plate.

\section{Measurements and data analysis}

A pressure sensor (CPCL04D, Honeywell) was utilized to measure the pressure difference between the top and bottom chambers. A bidirectional miniature flow meter (HAF-BLF0050, Honeywell) was used to measure the air flow moving into or out of the channels. Images of the thin film deformed at different voltages were taken with a digital camera (EO-1312C, Edmund Optics Inc.) at a rate of $10 \mathrm{fps}$ while a green laser line (LC532-5$3 \mathrm{~F}, 532 \mathrm{~nm} / 5 \mathrm{~mW}$ ) indicated a desired cross section of the thin film. A LabVIEW code was developed to generate proper signals for controlling the high voltage amplifier (Trek20/20C, Trek Inc.). In addition, the code was able to sync and trigger the camera while reading the output voltages of the power supply, pressure sensor, and flow meters. We also developed a MATLAB code and used the Image Processing Toolbox for extracting curvatures, creating 3D meshes, and calculating the geometrical flow rate, as indicated in the main text.

\section{Acknowledgements}

This work was supported by the Army Research Office Multidisciplinary University Research Initiative (ARO/MURI) under Grant no. W911NF-09-1-0476. M. Bozlar was partially funded by the Partner University Fund of the French American Cultural Exchange (PUF/FACE), sponsored by the French Embassy in the United States.

\section{Notes and references}

$\dagger$ To consider nonlinear terms, one can use the Helmholtz free energy along with the neo-Hookean model to obtain the equation of state for equal-biaxial prestretching condition $-\sigma_{\mathrm{i}}+\varepsilon \varepsilon_{0}\left(\frac{V}{h_{\mathrm{d}}}\right)^{2}=\frac{E}{2(1+\nu)}\left(\lambda^{2}-\lambda^{-4}\right),{ }^{20}$ where $\lambda=1+e_{\mathrm{r}}$ is the total film stretch, and the changes in the film thickness caused by the electric field are neglected. Using the stress-stretch relation in the neo-Hookean model, $\sigma_{\mathrm{r}}=\frac{E}{2(1+v)}\left(\lambda^{2}-\lambda^{-4}\right)$, and substituting the critical buckling stress $\left(\sigma_{\mathrm{r}}\right)_{\mathrm{cr}}$, we find an equation for $V_{\mathrm{c}}$ that is very similar to eqn (2).

1 T. Thorsen, S. Maerkl and S. Quake, Science, 2002, 298, 580584.

2 H. A. Stone, A. D. Stroock and A. Ajdari, Annu. Rev. Fluid Mech., 2004, 36, 381-411.

3 P. Tabeling, Introduction to Microfluidics, Oxford University Press, 2005. 
4 D. C. Leslie, C. J. Easley, E. Seker, J. M. Karlinsey, M. Utz, M. R. Begley and J. P. Landers, Nat. Phys., 2009, 5, 231-235.

5 D. P. Holmes, B. Tavakol, G. Froehlicher and H. A. Stone, Soft Matter, 2013, 9, 7049-7053.

6 J. C. Eijkel and A. Van Den Berg, Microfluid. Nanofluid., 2005, 1, 249-267.

7 R. B. Schoch, J. Han and P. Renaud, Rev. Mod. Phys., 2008, 80, 839.

8 T. Fujii, Microelectron. Eng., 2002, 61, 907-914.

$9 \mathrm{H}$. Andersson and A. Van den Berg, Sens. Actuators, B, 2003, 92, 315-325.

10 J. O. Tegenfeldt, C. Prinz, H. Cao, R. L. Huang, R. H. Austin, S. Y. Chou, E. C. Cox and J. C. Sturm, Anal. Bioanal. Chem., 2004, 378, 1678-1692.

11 R. Fair, Microfluid. Nanofluid., 2007, 3, 245-281.

12 M. Abdelgawad and A. R. Wheeler, Adv. Mater., 2009, 21, 920-925.

13 G. M. Whitesides, Lab Chip, 2011, 11, 191.

14 W. Wu, A. DeConinck and J. A. Lewis, Adv. Mater., 2011, 23, H178-H183.

15 F. Carpi, S. Bauer and D. De Rossi, Science, 2010, 330, 17591761.

16 R. E. Pelrine, R. D. Kornbluh and J. P. Joseph, Sens. Actuators, A, 1998, 64, 77-85.
17 R. Pelrine, R. Kornbluh, Q. Pei and J. Joseph, Science, 2000, 287, 836-839.

18 Z. Suo, MRS Bull., 2012, 37, 218-225.

19 M. Bozlar, C. Punckt, S. Korkut, J. Zhu, C. C. Foo, Z. Suo and I. A. Aksay, Appl. Phys. Lett., 2012, 101, 091907.

20 T. Lu, J. Huang, C. Jordi, G. Kovacs, R. Huang, D. R. Clarke and Z. Suo, Soft Matter, 2012, 8, 6167-6173.

21 J.-S. Plante and S. Dubowsky, Int. J. Solids Struct., 2006, 43, 7727-7751.

22 X. Zhao and Z. Suo, Phys. Rev. Lett., 2010, 104, 178302.

23 Z. Suo, Acta Mech. Solida Sin., 2010, 23, 549-578.

24 B. Li, H. Chen, J. Qiang, S. Hu, Z. Zhu and Y. Wang, J. Phys. D: Appl. Phys., 2011, 44, 155301.

25 R. Shankar, T. K. Ghosh and R. J. Spontak, Soft Matter, 2007, 3, 1116-1129.

26 P. Bulson, The Stability of Flat Plates, Chatto and Windus, 1970.

27 S. Rosset, M. Niklaus, P. Dubois and H. R. Shea, Sens. Actuators, A, 2008, 144, 185-193.

28 V. Rocco, F. Antonio, B. Massimo, C. Federico, F. Gabriele and R. Danilo De, Smart Mater. Struct., 2012, 21, 094005.

29 S. Rosset, M. Niklaus, P. Dubois and H. R. Shea, J. Microelectromech. Syst., 2009, 18, 1300-1308.

30 M. W. Westneat, J. J. Socha and W.-K. Lee, Annu. Rev. Physiol., 2008, 70, 119-142. 